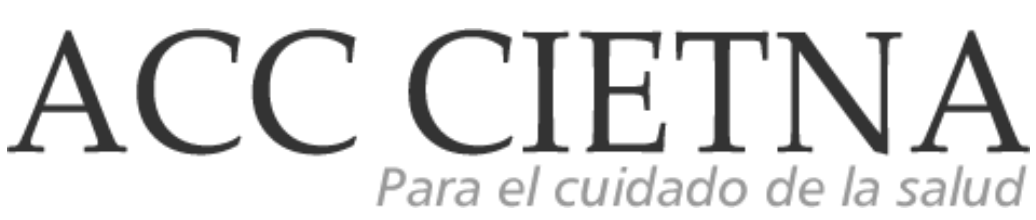

\title{
Estrés parental y sus dimensiones en Unidades de Cuidado Intensivo Neonatal - Pediátrica: Revisión Narrativa
}

\section{Parental stress and its dimensions in Neonatal - Pediatric Intensive Care Units: Narrative Review}

Beatriz Peña-Silva *, 1, a

beatriz.pena@uv.cl

https://orcid.org/0000-0001-9429-7551

\author{
Alejandro García-Araya ${ }^{1, ~ b}$ \\ alejandro.garciaa@alumnos.uv.cl \\ https://orcid.org/0000-0001-5888-0870
}

\section{Mayra Miranda-Iglesias ${ }^{1, b}$ \\ mayra.miranda@alumnos.uv.cl \\ https://orcid.org/0000-0001-8449-122X}

José Caviedes-Fernández ${ }^{1, ~ b}$

jose.caviedes@alumnos.uv.cl

https://orcid.org/0000-0002-8950-4218

\author{
Valeria Ulloa-Ramírez ${ }^{1, b}$ \\ valeria.ulloa@alumnos.uv.cl \\ https://orcid.org/0000-0002-9532-5021
}

\author{
Yuyunisse Rementería-Rementería 1,b \\ yuyunisse.rementeria@alumnos.uv.cl \\ https://orcid.org/0000-0002-2024-1464
}

Franco Loo-Allamand $1, b$

franco.loo@alumnos.uv.cl

https://orcid.org/0000-0001-6231-3262

\footnotetext{
* Autor corresponsal

1 Universidad de Valparaíso, Valparaíso, Chile

a Magister en Educación en Ciencias de la Salud

b Licenciado en enfermería
}

Fechas importantes

Recibido: 2021-06-07

Aceptado: 2021-11-15

Publicado online: 2021-12-17

\begin{abstract}
Resumen
La hospitalización de un menor es un evento estresante para todo el grupo familiar, donde los padres se ven más afectados debido a la alteración de su rol. Objetivo: Determinar el nivel de estrés y dimensiones que más afectan a los padres de menores hospitalizados en Unidades de Cuidados Intensivos neonatales-pediátricos y la descripción de intervenciones para su disminución. Método: La búsqueda bibliográfica se realizó en las bases de datos Web of Science, Scopus, Biblioteca Virtual en Salud, EBSCO y SciELO. Los descriptores utilizados fueron "estrés psicológico", "unidades de cuidado intensivo" y "padres". Inicialmente se seleccionaron 250 artículos, de los cuales fueron descartados por criterios de exclusión 214. Tras la lectura crítica de 35 artículos finalmente se seleccionaron 17. Resultados principales: La experiencia de la hospitalización de un hijo o hija en estas unidades es estresante para los padres, donde se visualiza mayoritariamente un moderado nivel de estrés en los padres, donde la diferencia entre sexos no es significativa. Las dimensiones más estresantes fueron la alteración del rol parental y apariencia y comportamiento del bebé, encontrando intervenciones exitosas
\end{abstract}


para disminuir el estrés parental. Conclusiones generales: Existe escasez de investigaciones en Unidades de Cuidados Intensivos pediátricos, siendo interesante estudiar en profundidad las características culturales en el estrés parental. El profesional de enfermería debe asumir como parte de sus labores el cuidado de los padres, a través de intervenciones como la comunicación empática y favoreciendo su participación activa, permitiendo que los padres puedan participar en algunos cuidados de su hijo o hija.

Palabras clave: Estrés psicológico; unidad de cuidados intensivos; padres; pediatría; hospitalización.

\section{Abstract}

The hospitalization of a minor is a stressful event for the entire family group, where parents are more affected due to the alteration of their role. Objective: To determine the level of stress and dimensions that most affect the parents of minors hospitalized in Units of Neonatal-Pediatric Intensive Care and the description of interventions for its reduction. Method: The bibliographic search was carried out in the Web of Science, Scopus, Virtual Health Library, EBSCO and SciELO databases. The descriptors used were "psychological stress", "intensive care units" and "parents". Initially, 250 articles were selected, of which 214 were discarded by exclusion criteria. After critical reading of 35 articles, 17 were finally selected. Main Results: The experience of hospitalization of a son or daughter in these units is stressful for the parents. where a moderate level of stress is mostly seen in the parents, where the difference between the sexes is not significant. The most stressful dimensions were the alteration of the parental role and appearance and behavior of the baby, finding successful interventions to reduce parental stress. General Conclusions: there is a shortage of research in Pediatric Intensive Care Unit, being interesting to study in depth the cultural characteristics of parental stress. The nursing professional must take care of the parents as part of their work, through interventions such as empathic communication and favoring their active participation, allowing parents to participate in some care of their son or daughter.

Keywords: Psychological stress; intensive care units; parents; pediatrics; hospitalization.

\section{Introducción}

El estrés es la respuesta fisiológica del organismo ante un estímulo externo, el que se puede manifestar en diferentes escenarios ${ }^{1}$. En cambio, el estrés parental es la respuesta asociada a un evento adverso proveniente de un hijo o hija, puesto que el rol de la paternidad asume la responsabilidad de crianza y cuidado de un hijo o hija, donde los padres se ven enfrentados a distintos estímulos provenientes de las demandas del ciclo vital y de las crisis no normativas emergentes, que pueden desencadenar estrés en estos ${ }^{2,3,4}$.

Así entonces, al estrés provocado por el conocimiento de la enfermedad de un hijo o hija, se suma la hospitalización, provocando un evento crítico en la familia debido a que es inesperado y se ven sometidos a diferentes cambios en sus rutinas, iniciado con el cambio a un lugar desconocido como lo es el hospital, seguido por la separación física que impide a los padres puedan encargarse de los cuidados de su hijo o hija ${ }^{5}$.

Es posible comprender que el proceso de hospitalización de un menor es un evento que genera una crisis en el grupo familiar, donde los padres se ven más afectados ya que altera y limita su rol, generando angustia ante la separación y el sentimiento de incapacidad de proteger a un hijo o hija en su situación de salud actual. Lo anterior se ve potenciado si la hospitalización se realiza en una Unidad de Cuidados Intensivos (UCI), considerando que el ingreso a estas unidades significa un estado lábil y complejo de salud que requiere de monitoreo permanente, así como tratamiento y cuidados de alta complejidad ${ }^{1,6}$.

En este contexto donde el padre o madre se ve separado del hijo o hija por la hospitalización, se pueden generar emociones como desesperación, miedo, impotencia, culpa, nostalgia y esperanza de su pronta recuperación ${ }^{1,6}$. 
Los padres se ven enfrentados a diferentes estímulos a raíz de la enfermedad y hospitalización de su hijo o hija, los que se pueden ver agrupados en cuatro áreas: el proveniente del menor, es decir, los signos y síntomas de la enfermedad, la apariencia y la presencia de invasivos o contenciones físicas. Por otra parte, está el área proveniente de los padres debido a la separación del menor y la entrega de los cuidados de este a un otro, viéndose modificado el rol como padres. El área del ambiente clínico, con elementos propios del hospital, como el gran número de personas, ruido ambiental y equipos hospitalarios, y, por último, lo relacionado al equipo de salud, por la difícil comunicación con la familia por parte del personal, debido a las explicaciones muy rápidas y el uso de lenguaje técnico ${ }^{5}$

Además, se ha observado que las emociones negativas que genera el estrés de los padres también afectan en su habilidad de aprendizaje, lo que podría interferir en su capacidad de entregar o colaborar en algunos cuidados adaptados a la actual situación de salud del menor y dificultar su comprensión sobre las dinámicas internas en estas unidades ${ }^{7}$.

La Organización Mundial de la Salud (OMS) estima que 5,6 millones de niños murieron antes de cumplir cinco años el 2016, lo que se traduce en 15.000 muertes de menores de este grupo etario por día ${ }^{8}$. Por otra parte, la mortalidad infantil en las Unidades de Cuidados Intensivos Pediátricos (UCIP) en Latinoamérica alcanzó un 13,29\%, mientras que en Europa fue de un $5 \%$, entre el 2008 y el $2018^{9}$.

Respecto a Chile, el 2019 las defunciones pediátricas, en el grupo etario de 0 a 14 años, corresponden a un total de 1.977 decesos $^{10}$. Bajo estas cifras y para dar respuesta a la demanda en las UCIP, en Chile la dotación de camas para estas unidades está determinada por lo propuesto por la Sociedad Española de Cuidados Intensivos Pediátricos, quienes establecen que se debe destinar entre un 4 a $8 \%$ de las camas pediátricas totales ${ }^{11}$.

Las investigaciones centradas en el estudio del estrés parental en la UCIP se remontan a la segunda mitad de la década de los ochenta, inicialmente en Estados Unidos (EE. UU.), donde Carter y Miles, enfermeras estadounidenses, desarrollan en 1985 el instrumento "Parental Stressor Scale: Paediatric Intensive Care Unit (PSS: PICU)", para poder medir el estrés de los padres de menores hospitalizados en UCIP ${ }^{12}$. Posteriormente Miles y Funks en 1998 desarrollaron el "Parental Stressor Scale: Neonatal Intensive Care Unit (PSS: NICU)", otra adaptación del PSS: PICU, pero específico para las Unidades de Cuidados Intensivos Neonatales (UCIN) y que se centra más en medir el estrés que genera el ambiente de estas unidades en los padres ${ }^{13}$. Otro instrumento existente actualmente para medir el estrés parental es uno basado en la teoría de Callista Roy el cual incluye las subescalas de estímulos focales con circunstancias del hijo, su estado de salud y estímulos contextuales, pero no será incluido en esta revisión ${ }^{14}$.

El profesional de enfermería se encuentra en todo el proceso hospitalario y es el encargado de valorar y acompañar en la adaptación de los menores y sus familias. Además, en las UCIP y UCIN cuenta con las habilidades cognitivas, técnicas y psicológicas que le permiten otorgar a los niños y niñas cuidados continuos y del más alto nivel, debido a la condición crítica en que éstos se encuentran, pero en esta instancia también es importante valorar los estímulos provenientes de la hospitalización, ya que estos repercuten en los padres, generando un proceso adaptativo, que, en el caso de ser ineficaz, la respuesta se manifestará como estrés parental ${ }^{15}$.

Sin embargo, el perfil de enfermería descrito en la actualidad está más asociado a sus capacidades técnicas, las que son insuficientes para cubrir todas las demandas que son 
necesarias en la unidad, sobre todo para poder acoger y apoyar a los familiares de los niños hospitalizados en estado de mayor cuidado ${ }^{9}$.

Debido a esto es de suma importancia la identificación y priorización de los factores que influyen como estresores parentales, para poder establecer nuevas estrategias para prevenir e intervenir de forma oportuna, entregando un cuidado biopsicosocial al menor y su familia para favorecer la adaptación y experiencia hospitalaria ${ }^{16}$.

Al interior del equipo de salud, el profesional enfermero/a posee un rol fundamental en la gestión de los cuidados, por esto se espera que el conocimiento del estrés parental existente y las áreas que influyen mayoritariamente en ellos en forma priorizada, entregue valiosa información que oriente en la generación de normativa para el quehacer de enfermería y del equipo de salud, además de mejorar la organización de los sistemas de trabajo y la elaboración de nuevas estrategias que consideren a los padres durante el proceso de hospitalización de su hijo o hija.

Así también, conocer los factores estresores predominantes en estas unidades permite intervenir de manera focalizada, intentando disminuir los efectos del ambiente clínico de las UCIP y UCIN sobre los padres, como los sonidos, el aspecto de la unidad, la apariencia y conducta del hijo o hija, y mejorar la comunicación de estos con el equipo de salud ${ }^{17}$.

Debido a los aspectos planteados anteriormente, los objetivos de este artículo de revisión es determinar el nivel de estrés y las dimensiones que más afectan a los padres de menores hospitalizados en Unidades de Cuidados Intensivos Neonatales-Pediátricos y describir las intervenciones para la disminución del estrés parental.

\section{Material y métodos}

Se realizó una revisión de tipo narrativa, en búsqueda de literatura científica en las bases de datos Web of Science (WOS), Scopus, Biblioteca Virtual en Salud (BVS), EBSCO y SciELO, utilizando los descriptores en ciencias de la salud (DeCS): "parents", "psychological stress" y "intensive care unit" en inglés, y "padres", "estrés psicológico" y "unidades de cuidado intensivo" en español. Se diseñaron ecuaciones de búsqueda con el operador booleano AND $18,19,20,21,22,23$

La búsqueda se realizó entre los meses de abril y octubre del 2020. Los criterios de inclusión fueron artículos de investigación original de tipo cuantitativa, cualitativa y mixta, publicados en los últimos 5 años (2016-2020), en idioma español e inglés, utilización de instrumento PSS: PICU o NICU, con sujetos de estudio los padres de menores hospitalizados en UCIP y/o UCIN. Por otra parte, los criterios de exclusión fueron artículos de revisión y reflexión, estudios que abordaron el estrés parental fuera del periodo de hospitalización, centrado en la patología del menor, en grupos específicos o que se realizaron en otras unidades clínicas.

Las etapas desarrolladas fueron las siguientes: planteamiento de la pregunta y objetivos del trabajo, búsqueda en la literatura de la información a partir de buscadores y bases de datos electrónicas, análisis de la literatura y redacción de los resultados.

Para la recolección de los datos se utilizó un instrumento de elaboración propia en Microsoft Excel que incluyó los siguientes ítems: base de datos, nombre del artículo, cumplimento de criterios de inclusión, año de publicación, tipo de estudio, unidad clínica donde se desarrolló la investigación, instrumento utilizado, muestra seleccionada, tipo de análisis, comité de ética 
que avala el estudio, lugar de procedencia del artículo, profesionales que abarca, principales resultados y conclusiones que aportan a responder los objetivos planteados en la revisión.

Para la selección de artículos en las bases de datos ya expuestas, en primera instancia se utilizaron los descriptores mencionados obteniendo 903 investigaciones. Al ingresar los filtros de años e idioma se obtuvieron 250 estudios. Luego, se excluyeron los artículos según revisión de título y verificando que estos fueran acorde a los objetivos planteados, quedando 71 investigaciones. Posteriormente, se descartaron artículos según la revisión de los resúmenes y presencia de criterios de exclusión ya descritos, obteniendo 50 publicaciones seleccionadas. A continuación, se eliminaron 15 estudios que se encontraban repetidos, lo que dejó un total de 35 investigaciones. Finalmente se realizó una lectura crítica del texto completo de las publicaciones preseleccionadas usando como criterios de exclusión el incumplimiento de los criterios CASPe para las investigaciones cualitativas y para los estudios cuantitativos se verificó la consistencia de la metodología, también se usó como criterio de exclusión para todos los artículos aquellos que no cumplieran con los criterios ético-legales y que los instrumentos utilizados no estuvieran validados ${ }^{24}$. Todos estos elementos dieron como resultado la eliminación de 18 publicaciones, quedando un total de 17 artículos cuantitativos para la revisión narrativa.

\section{Figura $\mathrm{N}^{\circ} 01$ \\ Flujograma de Metodología de Selección de Artículos}

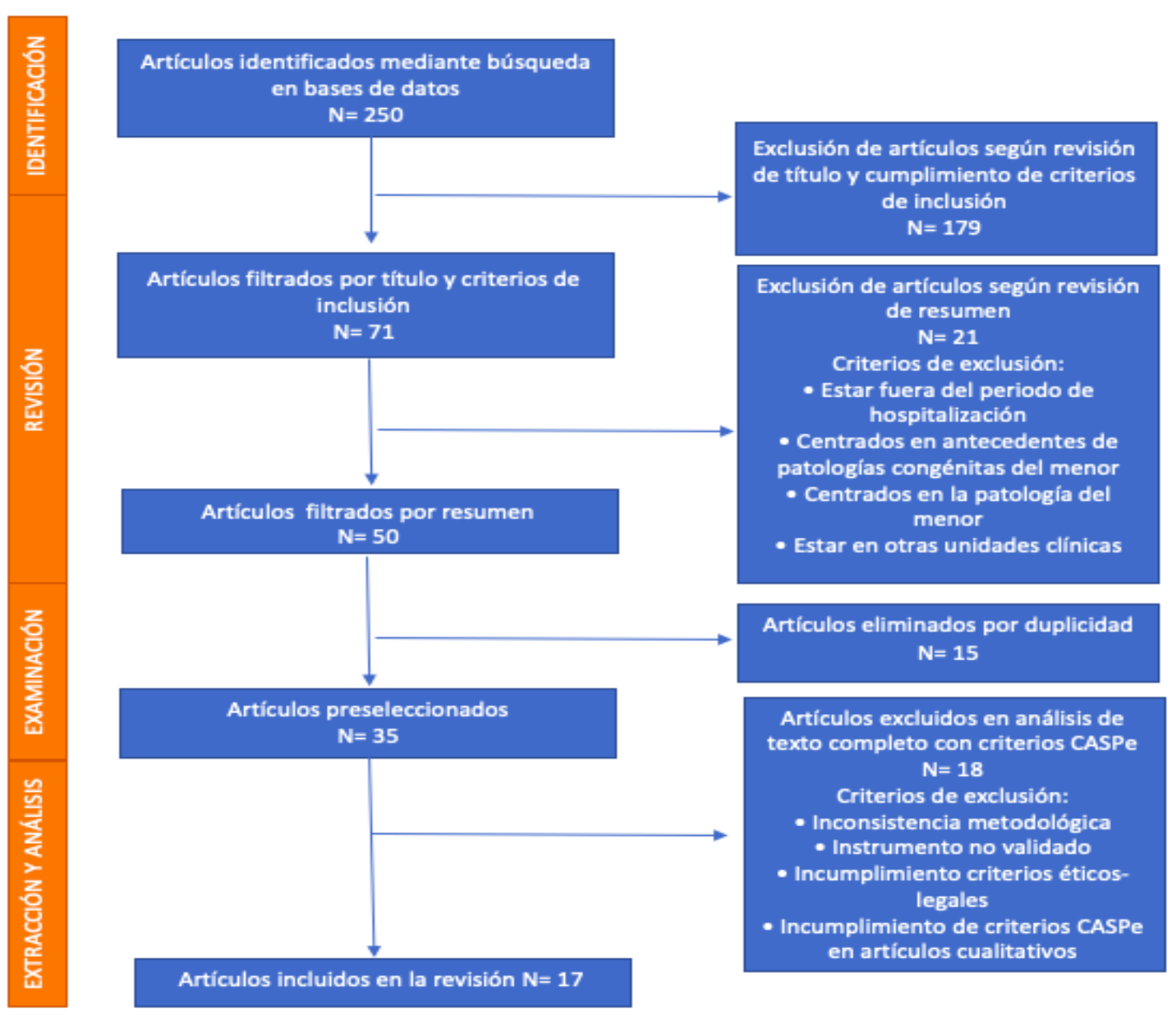




\section{Resultados y discusión}

Se presentan siguiendo una secuencia lógica, de ser necesario se pueden insertar tablas y figuras estrictamente necesarias siguiendo las formalidades APA. La revisión permitió localizar 17 artículos, los cuales se encontraron en las bases de datos mencionadas en la tabla 1, donde se destaca como principal fuente las bases de datos EBSCO y BVS.

Tabla $N^{\circ} 01$

Artículos en bases de datos

\begin{tabular}{|c|c|}
\hline Bases de datos & No de artículos \\
\hline BVS & 5 \\
\hline EBSCO & 5 \\
\hline SCIELO & 1 \\
\hline SCOPUS & 4 \\
\hline WOS & 2 \\
\hline TOTAL & $\mathbf{1 7}$ \\
\hline
\end{tabular}

Como se muestra en la tabla 2, el 52,9\% de las publicaciones se concentran en el año 2019, donde destaca Latinoamérica con el 35,2\% de los artículos, siendo los países que más aportan Brasil en primer lugar, seguido por México. Además, se puede observar que en el resto de los continentes el número de publicaciones es similar.

Tabla $\mathbf{N}^{\circ} 02$

Distribución por país de origen

\begin{tabular}{|l|l|c|c|}
\hline \multicolumn{2}{|c|}{ Localidad } & No de artículos & Años \\
\hline Europa & Alemania & 1 & 2017 \\
& Dinamarca & 1 & 2018 \\
& Noruega & 1 & 2019 \\
& Polonia & 1 & 2019 \\
& Suecia & 1 & 2016 \\
\hline Asia & Irán & 1 & 2016 \\
& Malasia & 1 & 2019 \\
& Turquía & 3 & $2018(2)-2019$ \\
\hline Latinoamérica & Brasil & 3 & $2018-2019(2)$ \\
& México & 2 & $2016-2019$ \\
& Chile & 2 & 2019 \\
\hline
\end{tabular}

Todos los estudios seleccionados son de tipo cuantitativo, siendo mixto uno de ellos, que se realizaron en UCIN y solo uno fue realizado de forma paralela en una UCIP $25,26,27,28,29,30,31,32,33,34,35,36,37,38,39,40,41$. Por consiguiente, el instrumento más utilizado para medir el estrés parental fue el Parental Stressor Scale: Neonatal Intensive Care Unit (PSS:NICU) con un $94,1 \%$ con sus respectivas adaptaciones para cada país, el cual es una variante específica del Parental Stressor Scale (PSS) para ser aplicada en UCIN, desarrollada por Miles y Funk ${ }^{13}$. Solo una publicación del año 2019 utilizó una versión de PSS en unidad Pediátrica ${ }^{12}$. 
Un $64.7 \%$ de las publicaciones contempla el estrés de ambos padres. Sin embargo, el 29,5\% de los estudios solo se enfocó en el estrés maternal, frente al 5,8\% que se centró en el estrés vivenciado por los padres durante el proceso de hospitalización de sus hijos, encontrando mayor interés por estudiar el estrés materno dentro del ámbito hospitalario. El 23,5\% de los estudios analizó la relación entre el nivel de estrés y el sexo de los padres, sin embargo, esta relación no fue estadísticamente significativa $25,26,27,28,29,30,31,32,33,34,35,36,37,38,39,40,41$.

Si bien la totalidad de artículos seleccionados realizaron una caracterización sociodemográfica de la muestra, ésta no estableció una relación con el estrés parental.

Dentro de los estudios se logra observar que el 64,7\% está enfocado hacia la práctica de enfermería, su papel dentro del proceso hospitalario de los menores y la importancia de su rol en la experiencia del suceso para los padres $25,27,29,31,32,33,34,35,36,39,40$. Por otro lado, el $29,4 \%$ de las publicaciones están enfocadas al equipo de salud en general y en cómo este influye en el proceso de hospitalización y la vivencia de los padres ${ }^{26,28,30,38,41}$. El 5,8\% restante no menciona los profesionales de salud ${ }^{37}$.

\section{Estrés Parental Global}

Del $70,5 \%$ de las investigaciones que reportaron el estrés global de los padres, utilizando el PSS:NICU, se encontró un $50 \%$ de estudios en Europa donde el promedio presentó estrés moderado y el otro $50 \%$ poco estresante $26,27,28,29,30$. Por otra parte, en Asia se evidenció en un $75 \%$ de los artículos que los padres presentaron estrés moderado y un $25 \%$ demostraron estrés alto $32,33,34,35$. A nivel Latinoamericano, un $50 \%$ de las publicaciones obtuvo un nivel moderado de estrés en los padres, $25 \%$ muy estresante y $25 \%$ poco estresante $36,37,38,39,40$. Mientras en Chile un $50 \%$ presentó estrés extremo y un $50 \%$ moderadamente estresante 25,41 .

\section{Estrés Parental por Dimensiones}

Respecto a las dimensiones que presentaron mayor estrés, el total de los artículos en Europa identificaron la alteración del rol parental como la dimensión más estresante 26,27,28,29,30. Mientras que en Asia el $66,6 \%$ de los artículos señalaron que los padres se ven más afectados por la apariencia y comportamiento del bebé, seguido de vistas y sonido con un $16,6 \%$ $31,32,33,34,35$. En Latinoamérica el $50 \%$ de la evidencia señaló que los padres se ven más afectados en la dimensión alteración del rol parental, seguido por un 37,5\% en la apariencia y comportamiento del bebé y en un $12,5 \%$ en la comunicación con el personal $36,37,38,39,40$. Por último, en Chile ambos artículos concuerdan que la dimensión más estresante fue la alteración del rol parental, seguida por comunicación con el personal ${ }^{25,41}$.

Tabla No 03

\section{Características de los artículos seleccionados}

\begin{tabular}{|c|c|c|c|}
\hline $\begin{array}{l}\text { Título del } \\
\text { estudio / } \\
\text { Lugar }\end{array}$ & $\begin{array}{l}\text { Metodología / } \\
\text { Instrumento }\end{array}$ & $\begin{array}{c}\text { Objetivo / } \\
\text { Muestra }\end{array}$ & Principales resultados \\
\hline $\begin{array}{l}\text { Estrés } \\
\text { experimentado } \\
\text { por madres de } \\
\text { recién nacidos } \\
\text { pre-término en } \\
\text { unidad de } \\
\text { terapia }\end{array}$ & $\begin{array}{l}\text { Cuantitativo, } \\
\text { transversal } \\
\text { Instrumento: } \\
\text { PSS:NICU }\end{array}$ & $\begin{array}{l}\text { Evaluar el nivel de } \\
\text { estrés de madres } \\
\text { de recién nacidos } \\
\text { prematuros con } \\
\text { edad gestacional } \\
\leq 34 \text { semanas, } \\
\text { internados en }\end{array}$ & $\begin{array}{l}\text { Las madres se encontraban en } \\
\text { una situación muy estresante. La } \\
\text { dimensión más estresante fue } \\
\text { "Cambios en el rol parental" con } \\
\text { un promedio de } 4.36 \text {, seguido } \\
\text { por "apariencia y } \\
\text { comportamiento del bebé" con }\end{array}$ \\
\hline
\end{tabular}




\begin{tabular}{|c|c|c|c|}
\hline $\begin{array}{l}\text { intensiva } \\
\text { neonatal } \\
\text { /Brasil }\end{array}$ & & $\begin{array}{l}\text { terapia intensiva } \\
\text { neonatal. } \\
74 \text { madres de } \\
\text { recién nacidos } \\
\text { prematuros en } \\
\text { UCIN. }\end{array}$ & $\begin{array}{l}3.32 \text { y finalmente la dimensión } \\
\text { "luces y sonidos" con un } \\
\text { promedio de } 2.80 \text {. Además, las } \\
\text { madres con experiencia previa } \\
\text { con prematuridad tenían un nivel } \\
\text { general de estrés más bajo que } \\
\text { aquellos sin experiencia. }\end{array}$ \\
\hline $\begin{array}{l}\text { Adaptación } \\
\text { cultural y } \\
\text { validación de } \\
\text { Parental } \\
\text { Stressor Scale: } \\
\text { Neonatal } \\
\text { Intensive Care } \\
\text { Unit en padres } \\
\text { mexicanos } \\
\text { /México }\end{array}$ & $\begin{array}{l}\text { Cuantitativo, } \\
\text { transversal } \\
\text { descriptivo } \\
\text { Instrumento: } \\
\text { PSS:NICU }\end{array}$ & $\begin{array}{l}\text { Adaptar } \\
\text { culturalmente y } \\
\text { validar la Escala de } \\
\text { Estrés Parental: } \\
\text { Unidad de Cuidados } \\
\text { Intensivos } \\
\text { Neonatales (PSS: } \\
\text { NICU) para su } \\
\text { aplicación en un } \\
\text { grupo de madres y } \\
\text { padres mexicanos. } \\
130 \text { madres y } \\
\text { padres de recién } \\
\text { nacidos prematuros } \\
\text { hospitalizados en la } \\
\text { UCIN del Hospital } \\
\text { General Dr. Manuel } \\
\text { Gea González de la } \\
\text { Ciudad de México. }\end{array}$ & $\begin{array}{l}\text { El estrés global fue de } 2.48 \\
\text { considerándose poco estresante. } \\
\text { La dimensión más estresante fue } \\
\text { "alteración del rol parental" con } \\
\text { un promedio de } 3.24 \text {, seguido de } \\
\text { "apariencia y comportamiento } \\
\text { del bebé" con un promedio de } \\
2.29 \text { y finalmente "luces y } \\
\text { sonidos" con un promedio de } \\
1.96\end{array}$ \\
\hline $\begin{array}{l}\text { Estrés en } \\
\text { padres de } \\
\text { recién nacidos } \\
\text { hospitalizados } \\
\text { en una unidad } \\
\text { de paciente } \\
\text { crítico neonatal } \\
\text { /Chile }\end{array}$ & $\begin{array}{l}\text { Cuantitativo, } \\
\text { transversal } \\
\text { descriptivo } \\
\text { Instrumento: } \\
\text { PSS:NICU }\end{array}$ & $\begin{array}{l}\text { Conocer el nivel de } \\
\text { estrés de padres de } \\
\text { recién nacidos } \\
\text { hospitalizados en } \\
\text { una UPCN y su } \\
\text { asociación con } \\
\text { variables clínicas y } \\
\text { sociodemográficas. } \\
100 \text { padres (43 } \\
\text { padres y } 57 \\
\text { madres) de recién } \\
\text { nacidos } \\
\text { hospitalizados en } \\
\text { una clínica privada. }\end{array}$ & $\begin{array}{l}\text { El estrés global fue de } 3.59 \text {, } \\
\text { calificándose como } \\
\text { moderadamente estresante. } \\
\text { La dimensión más estresante fue } \\
\text { "relación con el bebé" y "rol } \\
\text { parental" con un promedio de } 4.2\end{array}$ \\
\hline $\begin{array}{l}\text { Stress in } \\
\text { Parents of } \\
\text { Newborns in a } \\
\text { Neonatal } \\
\text { Intensive Care } \\
\text { Unit /Brasil }\end{array}$ & $\begin{array}{l}\text { Cuantitativo, } \\
\text { descriptivo } \\
\text { Instrumento: } \\
\text { PSS:NICU }\end{array}$ & $\begin{array}{l}\text { Identificar el nivel } \\
\text { de estrés y las } \\
\text { situaciones más } \\
\text { estresantes para los } \\
\text { padres de recién } \\
\text { nacidos internados } \\
\text { en una UCIN. } \\
204 \text { Padres (127 } \\
\text { mamás y } 77 \text { papás) }\end{array}$ & $\begin{array}{l}\text { El promedio de estrés global fue } \\
\text { de } 2.86 \text {, calificándose como } \\
\text { moderadamente estresante. La } \\
\text { dimensión que generó mayor } \\
\text { estrés fue "alteración del rol } \\
\text { parental" con } 3.49 \text {, donde } \\
\text { destacan "Estar separada(o) de } \\
\text { mi bebé" (promedio=4,00) y } \\
\text { "Sentirse desamparada y incapaz } \\
\text { de proteger a mi bebé del dolor y }\end{array}$ \\
\hline
\end{tabular}




\begin{tabular}{|c|c|c|c|}
\hline & & & $\begin{array}{l}\text { de los procedimientos } \\
\text { dolorosos". } \\
\text { En la dimensión "apariencia" y } \\
\text { "comportamiento del bebé" se } \\
\text { obtuvo un promedio de } 2.83 \text {. La } \\
\text { dimensión que presentó menor } \\
\text { estrés fue "imágenes y sonidos" } \\
\text { con un promedio de } 2.26\end{array}$ \\
\hline $\begin{array}{l}\text { Polish version } \\
\text { of the parental } \\
\text { stressor scale: } \\
\text { Neonatal } \\
\text { intensive care } \\
\text { unit } \\
\text { /Polonia }\end{array}$ & $\begin{array}{l}\text { Cuantitativo, } \\
\text { descriptivo } \\
\text { Instrumento: } \\
\text { PSS:NICU }\end{array}$ & $\begin{array}{l}\text { Validar la escala } \\
\text { PSS:NICU para la } \\
\text { población polaca. } \\
151 \text { padres de } \\
\text { infantes } \\
\text { prematuros } r \\
\text { madres y } 22 \\
\text { padres) mayores de } \\
18 \text { años }\end{array}$ & $\begin{array}{l}\text { El estrés global promedio fue } \\
\text { moderado con un 2,68. La } \\
\text { dimensión más alterada fue } \\
\text { "Alteración del rol parental" con } \\
\text { un 3,46, seguida por "luces y } \\
\text { sonidos con un 2,68. La } \\
\text { dimensión menos estresante fue } \\
\text { "Apariencia del bebé" con 2,33. }\end{array}$ \\
\hline $\begin{array}{l}\text { Stress level in } \\
\text { parents of } \\
\text { hospitalized } \\
\text { children in } \\
\text { pediatric and } \\
\text { neonatal } \\
\text { critical care } \\
\text { units / Chile }\end{array}$ & $\begin{array}{l}\text { Cuantitativo, } \\
\text { descriptivo } \\
\text { Instrumento: } \\
\text { PSS }\end{array}$ & 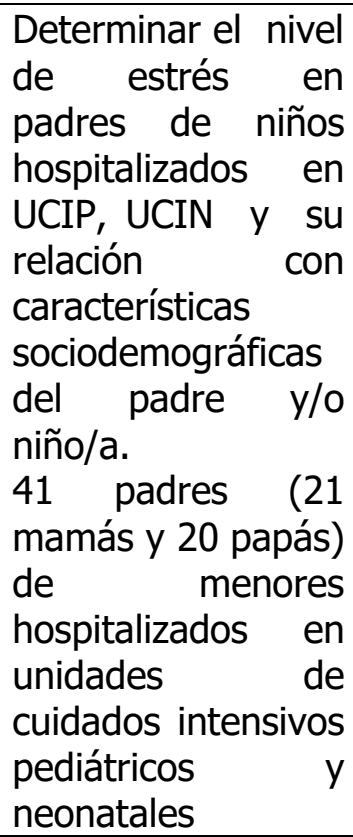 & $\begin{array}{l}\text { Los niveles de estrés global } \\
\text { fueron de un } 48.8 \% \text {, } \\
\text { extremadamente estresante, } \\
29.3 \% \text { muy estresante, } 12.2 \% \\
\text { moderadamente estresante y } \\
9.7 \% \text { poco o nada estresante. La } \\
\text { dimensión más estresante fue la } \\
\text { "alteración del rol parental" } \\
(3,38) \text {, seguido del "tratamiento } \\
\text { recibido" (3,20) y la } \\
\text { "comunicación con el personal" } \\
(3.03) \text {. }\end{array}$ \\
\hline $\begin{array}{l}\text { Parent } \\
\text { psychological } \\
\text { wellbeing in a } \\
\text { single-family } \\
\text { room versus an } \\
\text { open bay } \\
\text { neonatal } \\
\text { intensive care } \\
\text { unit / Noruega }\end{array}$ & $\begin{array}{l}\text { Cuantitativo, } \\
\text { prospectivo } \\
\text { Instrumento: } \\
\text { PSS:NICU }\end{array}$ & $\begin{array}{l}\text { Comparar los } \\
\text { niveles de estrés de } \\
\text { padres de recién } \\
\text { nacidos prematuros } \\
\text { extremos en una } \\
\text { sala individual y en } \\
\text { sala común } \\
132 \text { padres y } \\
\text { madres } \\
\text { prematuros de } \\
\text { extremos. }\end{array}$ & $\begin{array}{l}\text { "Luces y sonidos" fue la } \\
\text { dimensión más estresante con } \\
\text { un puntaje de } 2,2 \text {. La "Alteración } \\
\text { del rol parental" alcanzó un bajo } \\
\text { estrés con } 1,8 \text {. }\end{array}$ \\
\hline
\end{tabular}




\begin{tabular}{|c|c|c|c|}
\hline $\begin{array}{l}\text { Investigation } \\
\text { of stress and } \\
\text { nursing } \\
\text { support in } \\
\text { mothers of } \\
\text { preterm infants } \\
\text { in neonatal } \\
\text { intensive care } \\
\text { units. / Turquía }\end{array}$ & $\begin{array}{l}\text { Cuantitativo, } \\
\text { descriptivo } \\
\text { transversal } \\
\text { Instrumento: } \\
\text { PSS:NICU }\end{array}$ & $\begin{array}{l}\text { Determinar la } \\
\text { correlación entre el } \\
\text { estrés y niveles de } \\
\text { apoyo de los padres } \\
\text { con la edad y la } \\
\text { educación de las } \\
\text { madres estado, } \\
\text { número de hijos, } \\
\text { semana de } \\
\text { gestación del bebé } \\
\text { y el período de } \\
\text { hospitalización del } \\
\text { lactante entre las } \\
\text { madres de bebés } \\
\text { prematuros } \\
\text { hospitalizados en la } \\
\text { UCIN. } \\
106 \text { madres de } \\
\text { prematuros }\end{array}$ & $\begin{array}{l}\text { Se obtuvo un estrés global alto } \\
\text { de 2.92, las dimensiones más } \\
\text { estresantes fueron "vistas y } \\
\text { sonidos" y "comportamiento } \\
\text { infantil y apariencia". Además, } \\
\text { los niveles de estrés de las } \\
\text { madres con bebés conectados a } \\
\text { ventilación mecánica y } \\
\text { alimentados por vía parenteral } \\
\text { fueron altos. }\end{array}$ \\
\hline $\begin{array}{l}\text { The } \\
\text { effectiveness } \\
\text { of a structured } \\
\text { nursing } \\
\text { intervention } \\
\text { program on } \\
\text { maternal stress } \\
\text { and ability } \\
\text { among } \\
\text { mothers of } \\
\text { premature } \\
\text { infants in a } \\
\text { neonatal } \\
\text { intensive care } \\
\text { unit./ Malasia }\end{array}$ & $\begin{array}{l}\text { Cuantitativo, } \\
\text { cuasi- } \\
\text { experimental } \\
\text { Instrumento: } \\
\text { PSS:NICU }\end{array}$ & $\begin{array}{l}\text { Investigar el efecto } \\
\text { de una intervención } \\
\text { de enfermería } \\
\text { estructurada sobre } \\
\text { habilidades } \\
\text { parentales después } \\
\text { de la admisión de } \\
\text { infantes } \\
\text { prematuros } \\
\text { UCIN. en } \\
216 \quad \text { madres } \\
\text { mayores de 18 } \\
\text { años de prematuros }\end{array}$ & $\begin{array}{l}\text { El estrés global post intervención } \\
\text { fue de un promedio de } 3.50 \text { para } \\
\text { el grupo control y para el grupo } \\
\text { intervención fue de } 3.26 \\
\text { Posterior a la intervención la } \\
\text { dimensión más estresante para } \\
\text { el grupo control fue "alteración } \\
\text { del rol parental" con un promedio } \\
\text { de } 3.93 \text { y para el grupo } \\
\text { intervención fue igualmente } \\
\text { "alteración del rol parental" pero } \\
\text { con un promedio de } 3.46 \text {. }\end{array}$ \\
\hline $\begin{array}{l}\text { The stress } \\
\text { levels of } \\
\text { parents of } \\
\text { premature } \\
\text { infants and } \\
\text { related factors } \\
\text { in neonatal } \\
\text { intensive care } \\
\text { units / Turquía }\end{array}$ & $\begin{array}{l}\text { Cuantitativo, } \\
\text { descriptivo } \\
\text { Instrumento: } \\
\text { PSS:NICU }\end{array}$ & $\begin{array}{l}\text { Determinar los } \\
\text { niveles de estrés de } \\
\text { los padres cuyo } \\
\text { bebé prematuro } \\
\text { está hospitalizado } \\
\text { en UCIN y } \\
\text { determinar los } \\
\text { factores que } \\
\text { afectan su estrés. } \\
101 \text { madres y } \\
\text { padres de } \\
\text { prematuros en la } \\
\text { UCIN de un hospital } \\
\text { universitario. }\end{array}$ & $\begin{array}{l}\text { La puntuación global fue de } 3,23, \\
\text { no encontrando diferencia entre } \\
\text { madres y padres. } \\
\text { En las dimensiones "apariencia y } \\
\text { comportamiento del bebé" } \\
\text { alcanzó el más alto índice con } \\
3,53 ; \text { mientras en las "vistas y los } \\
\text { sonidos" se obtuvo un } 3,03 \text {. La } \\
\text { subescala de "rol de los padres" } \\
\text { fue de } 2,90 \text {. Además, los padres } \\
\text { de bebés conectados a equipos } \\
\text { respiratorios experimentaron un } \\
\text { mayor nivel de estrés }\end{array}$ \\
\hline $\begin{array}{l}\text { Fathers' Stress } \\
\text { in the Neonatal }\end{array}$ & $\begin{array}{l}\text { Cuantitativo, } \\
\text { descriptivo }\end{array}$ & $\begin{array}{l}\text { Investigar el efecto } \\
\text { de una UCIN más } \\
\text { amigable para los }\end{array}$ & $\begin{array}{l}\text { El análisis de las diferencias de } \\
\text { las puntuaciones de estrés desde } \\
\text { el momento de la admisión hasta }\end{array}$ \\
\hline
\end{tabular}




\begin{tabular}{|c|c|c|c|}
\hline $\begin{array}{l}\text { Intensive Care } \\
\text { Unit } \\
\text { / Dinamarca }\end{array}$ & $\begin{array}{l}\text { Instrumento: } \\
\text { PSS:NICU }\end{array}$ & 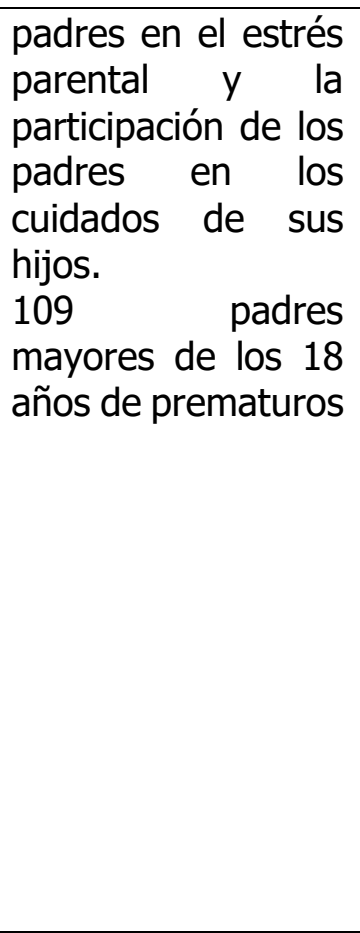 & $\begin{array}{l}\text { el momento del alta mostró que } \\
\text { para ambos grupos las } \\
\text { puntuaciones de estrés } \\
\text { disminuyeron significativamente } \\
\text { durante la hospitalización, } \\
\text { variando de un nivel poco } \\
\text { estresante a nada estresante. } \\
\text { La dimensión más estresante al } \\
\text { momento de la admisión fue el } \\
\text { "Rol Parental", tanto para el } \\
\text { grupo control (1.94) como para } \\
\text { el grupo intervención (2.10). } \\
\text { Para el momento del alta, se } \\
\text { observó que la dimensión más } \\
\text { estresante que generó mayor } \\
\text { estrés fue el "Rol Parental" en el } \\
\text { grupo control (1.50), en cambio, } \\
\text { para el grupo intervención fue la } \\
\text { "Apariencia y Comportamiento } \\
\text { del bebé" (1.87) }\end{array}$ \\
\hline $\begin{array}{lr}\text { The Effect of } \\
\text { Spiritual Care } \\
\text { on Stress } \\
\text { Levels } \\
\text { Mothers of } \\
\text { NICU. } \\
\text { / Turquía }\end{array}$ & $\begin{array}{l}\text { Cuantitativo, } \\
\text { correlacional } \\
\text { Instrumento: } \\
\text { PSS:NICU }\end{array}$ & $\begin{array}{l}\text { Identificar el efecto } \\
\text { de una intervención } \\
\text { espiritual sobre los } \\
\text { niveles de estrés de } \\
\text { madres de infantes } \\
\text { hospitalizados en } \\
\text { UCIN. } \\
62 \text { madres de } \\
\text { prematuros. }\end{array}$ & $\begin{array}{l}\text { El estrés global posterior a la } \\
\text { intervención espiritual fue de } \\
3.70 \text { versus el grupo control que } \\
\text { presentó un estrés promedio de } \\
3.97 \\
\text { La dimensión más estresante } \\
\text { para ambos grupos fue "luces y } \\
\text { sonidos", sin embargo, el grupo } \\
\text { control presentó un promedio de } \\
4.31 \text { y el grupo intervención un } \\
\text { promedio de } 4.05\end{array}$ \\
\hline $\begin{array}{l}\text { Communicatin } \\
\text { g with parents } \\
\text { in neonatal } \\
\text { intensive care } \\
\text { units: The } \\
\text { impact on } \\
\text { parental stress. } \\
\text { / Alemania }\end{array}$ & $\begin{array}{l}\text { Mixto } \\
\text { Instrumento: } \\
\text { PSS:NICU }\end{array}$ & $\begin{array}{l}\text { Analizar el estrés en } \\
\text { padres cuyos bebés } \\
\text { con muy bajo peso } \\
\text { al nacer acaban de } \\
\text { concluir nivel de } \\
\text { atención en una } \\
\text { Unidad de Cuidados } \\
\text { Intensivos } \\
\text { Neonatales. } \\
1277 \text { sobre el } \\
\text { cuidado de } 923 \\
\text { bebés de } 66 \text { UCIN } \\
\text { alemanas. }\end{array}$ & $\begin{array}{l}\text { La mayoría de los participantes } \\
\text { experimentaron niveles } \\
\text { relativamente bajos de estrés en } \\
\text { "comunicación" con el personal } \\
\text { clínico y un nivel moderado de } \\
\text { estrés causado por la alteración } \\
\text { del "rol parental". } \\
\text { La comunicación con el personal } \\
\text { clínico fue percibida como muy } \\
\text { empática por muchos pacientes, } \\
\text { existiendo asociaciones } \\
\text { negativas pequeñas a moderadas } \\
\text { entre la comunicación y estrés. } \\
\text { Después de la implementación } \\
\text { del SNI, la reducción del estrés } \\
\text { materno general reveló que las } \\
\text { estrategias y el apoyo fueron } \\
\text { efectivos para reducir el estrés } \\
\text { materno. }\end{array}$ \\
\hline
\end{tabular}




\begin{tabular}{|c|c|c|c|}
\hline & & & $\begin{array}{l}\text { Además, padres de una edad } \\
\text { más temprana y los de bebés con } \\
\text { pronósticos graves, tenían más } \\
\text { probabilidades de experimentar } \\
\text { estrés. }\end{array}$ \\
\hline $\begin{array}{lr}\text { Estrés } & \\
\text { percibido por } \\
\text { los padres } & \text { del } \\
\text { neonato en } \\
\text { estado crítico } \\
\text { durante } \\
\text { proceso el } \\
\text { hospitalización. } \\
\text { / México }\end{array}$ & $\begin{array}{l}\text { Cuantitativo, } \\
\text { descriptivo } \\
\text { Instrumento: } \\
\text { PSS:NICU }\end{array}$ & 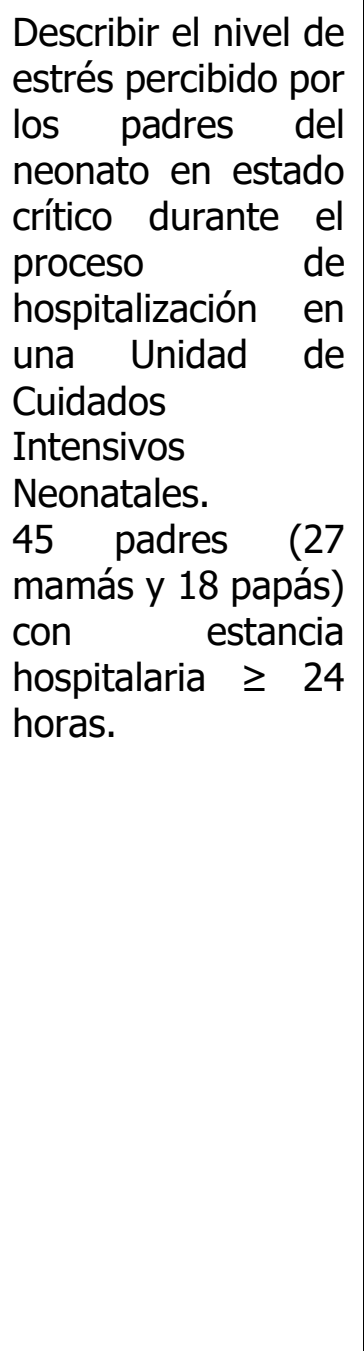 & $\begin{array}{l}\text { No estresante } 24 \% \text { Poco } \\
\text { estresante } 36 \quad \% \text {, } \\
\text { Moderadamente estresante } 25 \\
\% \text {, Muy estresante } 10 \% \\
\text { Extremadamente estresante } 5 \\
\% \text {. El nivel de estrés percibido } \\
\text { por los padres en los estresores } \\
\text { extrapersonales fue en su } \\
\text { mayoría poco estresante con } \\
\text { promedio mínimo de } 1.60 \text {, y } \\
\text { máximo de } 3.66 \text { en los "Aspectos } \\
\text { y sonidos de la unidad" y } \\
\text { "Apariencia y conducta del niño", } \\
\text { en los ítems de apariencia de las } \\
\text { heridas, cortadas o incisiones y } \\
\text { tubos. equipos en o cerca del } \\
\text { bebe fue percibido por los padres } \\
\text { como moderadamente } \\
\text { estresante. } \\
\text { El nivel de estrés de los padres } \\
\text { relacionado con los estresores } \\
\text { interpersonales "Relaciones y rol } \\
\text { de los padres" y "Comunicación } \\
\text { con el personal", fue poco } \\
\text { estresante con promedios entre } \\
2.0 \text { y } 2.62 \text { el nivel muy } \\
\text { estresante alcanzó promedios } \\
\text { entre } 4.0 \text { y } 4.17 \text { para el rubro de } \\
\text { "comunicación con el personal". }\end{array}$ \\
\hline $\begin{array}{l}\text { Evaluation of } \\
\text { the effect of } \\
\text { narrative } \\
\text { writing on the } \\
\text { stress sources } \\
\text { of the parents } \\
\text { of preterm } \\
\text { neonates } \\
\text { admitted to the } \\
\text { NICU / Irán }\end{array}$ & $\begin{array}{l}\text { Cuantitativo, } \\
\text { descriptivo } \\
\text { Instrumento: } \\
\text { PSS:NICU }\end{array}$ & $\begin{array}{l}\text { Identificar si la } \\
\text { compresión } \\
\text { enfermeras de } \\
\text { familiares sobre el } \\
\text { estrés parental } \\
\text { tiene impacto sobre } \\
\text { la satisfacción de } \\
\text { los padres. } \\
70 \text { madres de } \\
\text { recién nacidos } \\
\text { prematuros } \\
\text { hospitalizados en } 2 \\
\text { hospitales docentes } \\
\text { de Irán. }\end{array}$ & $\begin{array}{l}\text { La dimensión más estresante } \\
\text { para ambos grupos fue } \\
\text { "apariencia y comportamiento } \\
\text { del bebé" seguido de "alteración } \\
\text { del rol parental" y finalmente } \\
\text { "luces y sonidos". }\end{array}$ \\
\hline
\end{tabular}




\begin{tabular}{|c|c|c|c|}
\hline $\begin{array}{l}\text { Translation } \\
\text { and } \\
\text { psychometric } \\
\text { evaluation of a } \\
\text { Swedish } \\
\text { version of the } \\
\text { parental } \\
\text { stressor scale } \\
\text { PSS: NICU. } \\
\text { / Suecia }\end{array}$ & $\begin{array}{l}\text { Cuantitativo, } \\
\text { descriptivo } \\
\text { Instrumento: } \\
\text { PSS:NICU }\end{array}$ & $\begin{array}{l}\text { Traducir la escala } \\
\text { de estrés parental } \\
\text { PSS:NICU al idioma } \\
\text { sueco y evaluar las } \\
\text { propiedades } \\
\text { psicométricas de la } \\
\text { versión sueca. } \\
95 \text { padres } \\
\text { padres y } 34 \\
\text { madres) mayores } \\
\text { de } 18 \text { años, en } 2 \\
\text { UCIN de dos } \\
\text { hospitales } \\
\text { diferentes. }\end{array}$ & $\begin{array}{l}\text { Los padres experimentan } \\
\text { situaciones estresantes } \\
\text { particulares en la UCIN y un alto } \\
\text { nivel de estrés, experimentando } \\
\text { varios aspectos del entorno de la } \\
\text { UCIN. } \\
\text { En la subescala de "visores y } \\
\text { sonido", tener una máquina que } \\
\text { respire por mi bebé se consideró } \\
\text { estresante. }\end{array}$ \\
\hline $\begin{array}{l}\text { Measurement } \\
\text { of Family- } \\
\text { centered care } \\
\text { perception and } \\
\text { parental stress } \\
\text { in a neonatal } \\
\text { unit. / Brasil }\end{array}$ & $\begin{array}{l}\text { Cuantitativo, } \\
\text { descriptivo } \\
\text { cuasi- } \\
\text { experimental } \\
\text { Instrumento: } \\
\text { PSS:NICU }\end{array}$ & $\begin{array}{l}\text { Evaluar los efectos } \\
\text { de la } \\
\text { implementación del } \\
\text { modelo centrado en } \\
\text { la familia en la } \\
\text { salud y el estrés de } \\
\text { padres } \\
132 \text { padres (padre } \\
\text { y/o madre) }\end{array}$ & $\begin{array}{l}\text { El efecto de la intervención } \\
\text { realizada en este estudio } \\
\text { demostró una disminución en las } \\
\text { puntuaciones promedio de estrés } \\
\text { parental en los dominios de la } \\
\text { escala PSS: NICU, mostrando una } \\
\text { mayor caída en el dominio de } \\
\text { "Alteración del Rol Parental" (de } \\
4,2 \text { para 3,8), lo que representa } \\
\text { una disminución del estrés de } \\
\text { muy estresante en la fase previa } \\
\text { a la intervención hasta } \\
\text { moderadamente estresante en la } \\
\text { fase posterior a la intervención. } \\
\text { La dimensión que provocó mayor } \\
\text { estrés en los padres fue la } \\
\text { "Apariencia y Comportamiento } \\
\text { del bebé". }\end{array}$ \\
\hline
\end{tabular}

\section{Intervenciones y sus efectos en el Estrés Parental}

Es relevante mencionar que un $41,1 \%$ de las publicaciones realizaron una intervención para generar cambios en el nivel de estrés de los padres, donde se observó que en la mayoría hubo una variación positiva en los niveles de estrés global 26,27,30,31,33,34,38.

Una investigación realizada en Dinamarca donde se buscaba integrar a los padres en los cuidados de sus hijos en la UCIN demostró que a los padres que se les realizó la intervención presentaron mayor estrés que el grupo control, esto asociado a que el grupo intervención poseían más responsabilidades, generando una percepción de mayor presión por las expectativas sociales y del personal sobre su participación en el cuidado de su hijo/a26.

Otra publicación realizada en Noruega comparó dos salas de UCIN en donde se favoreció el acompañamiento, evidenciando que la presencia continua de los padres disminuye el estrés y el riesgo de depresión y angustia, pero no de forma significativa ${ }^{27}$.

Otra intervención en Alemania, donde el personal realizó a los padres una orientación durante el ingreso de sus hijos o hijas a la UCIN, concluyó que una comunicación empática desde el 
profesional a los padres genera un efecto positivo en el estrés parental ${ }^{30}$. De la misma forma, en Malasia se implementó una intervención similar por parte del equipo de salud que consistió en ocupar un programa que buscaba informar a la madre sobre todo el proceso de hospitalización de su hijo o hija. Se pudo concluir que esta intervención podría reducir significativamente el estrés materno y ayudar a mejorar las habilidades de las madres dentro de la UCIN ${ }^{34}$.

Por otro lado, un estudio en Turquía donde se realizó una intervención de cuidado espiritual a madres de recién nacidos logró observar diferencia en la dimensión apariencia y comportamientos del bebé a favor del grupo de la intervención espiritual, sin embargo, la relación tampoco fue estadísticamente significativa ${ }^{31}$.

También, en una investigación en Irán que realizó una comparación para evaluar la eficacia de la escritura narrativa para disminuir el estrés parental en las madres de niños hospitalizados en UCIN, determinó que la escritura narrativa disminuyó el estrés de forma estadísticamente significativa del grupo intervención en las 3 dimensiones del instrumento, obteniendo una disminución más importante en la dimensión apariencia y comportamiento del bebé ${ }^{33}$.

En cuanto a los estudios realizados en Latinoamérica, se observó en Brasil que la intervención del Programa de Implementación del Modelo del Cuidado Centrado en el Paciente y la Familia en la Unidad Neonatal (PIMCCPF-UN). Logró una disminución en el nivel de estrés general, especialmente en la dimensión rol parental, donde disminuyó el estrés desde muy estresante a un estrés moderado ${ }^{38}$.

De acuerdo a los resultados, se puede evidenciar que el proceso hospitalario en una UCI, independiente del país de origen de las personas que conformaron las muestras, genera estrés en alguna intensidad en los padres, lo que se explica según Astudillo et al., porque el proceso de hospitalización de un menor es un evento que genera una crisis no normativa en los padres e hijos, e inclusive, de todo el núcleo familiar, esto a raíz de la separación, la angustia y el sentimiento de incapacidad de protegerlos de su situación de salud actual ${ }^{1}$. A lo anterior, se suma lo señalado por Ramírez et al, donde se menciona la pérdida del rol parental como un factor determinante en el estrés parental en las UCIP, concordando con los resultados obtenidos en esta revisión bibliográfica ${ }^{5}$.

Si bien, la mayoría de las publicaciones estudian el estrés de ambos padres, existe contraste entre los resultados y la literatura existente. Según Sankar et al. ha descrito que las madres tienden a presentar mayores niveles de estrés y sintomatología ansiosa-depresiva ${ }^{42}$. Esto último Aguiñaga-Zamarripa et al. lo atribuye a que las madres al no poder entregar cuidados a sus hijos recién nacidos, no logran reconocerlos como sus propios bebés debido a la dificultad que esto supone para establecer un vínculo de apego entre ellos ${ }^{38}$. Sin embargo, a través de esta revisión se pudo notar que la diferencia entre sexos no es significativa, aunque debido a que la cantidad de artículos encontrados no fue muy abundante, esta última aseveración podría estar limitada.

Respecto a las diferencias encontradas en el estrés por dimensión en las distintas regiones (occidente y oriente), la literatura encontrada no realiza un mayor análisis del porqué de este fenómeno, sin embargo, las características culturales que existen entre occidente y oriente podrían ser parte de la justificación de las diferencias encontradas en las dimensiones.

En cuanto a lo observado en Chile, las diferencias en el nivel global de estrés se podrían explicar por la desigualdad de condiciones en los establecimientos, considerando que uno 
pertenece a la red de salud pública y el otro al sistema privado, sin embargo, esta información pierde fuerza si se considera que la dimensión donde se generó mayor estrés, en ambos estudios, fue la alteración del rol parental, lo que plantea la necesidad de nueva evidencia que asocie el nivel de estrés con los ámbitos que involucran los recursos disponibles, como la comunicación entre el equipo de salud con los padres de menores hospitalizados, en ambos tipos de establecimientos ${ }^{25,41}$. Una de las grandes diferencias que se pueden encontrar entre ambos centros es el ambiente en donde están inmersos, por un lado, en el espacio público los padres y sus hijos o hijas hospitalizados se encuentran en salas compartidas con más usuarios, mientras, en el sistema privado, por lo general, cuentan con salas individuales donde los padres pueden tener un ambiente de mayor intimidad y tranquilidad ${ }^{27}$.

Por otra parte, el estudio que presentó niveles de estrés extremo, evaluó a padres de menores en UCIP, lo que se puede explicar con lo inesperado que pueden ser las hospitalizaciones en niños y niñas de mayor edad, lo que en las UCIN no siempre está presente, ya que existe un número importante de padres que se encuentran informados y parcialmente preparados para la hospitalización de su hijo o hija al momento de nacer, por alguna enfermedad congénita o problema de salud que se haya detectado durante el embarazo ${ }^{1}$.

Respecto a las intervenciones, Doupnik et al en su estudio de meta-análisis señala que la educación a los padres sobre habilidades de cuidado e intervenciones centradas en el bienestar emocional de estos, como actividades de relajación, expresión emocional y manejo del estrés, generan un impacto positivo en sus niveles de estrés ${ }^{43}$. Lo anterior coincide con los hallazgos de esta revisión, reforzando la idea de que existen intervenciones efectivas que pueden disminuir los niveles de estrés en los padres cuyos hijos o hijas se encuentran hospitalizados en UCIN o UCIP.

Frente a los resultados planteados, el rol profesional del enfermero/a en unidades de paciente crítico neonatal o pediátricas, debe estar asociado íntimamente con la responsabilidad de entregar cuidados holísticos tanto a los menores como a sus padres, considerando factores psicológicos, espirituales y culturales, brindándoles herramientas necesarias para fomentar la cercanía, el contacto físico temprano y los cuidados básicos de su hijo o hija, ya que, según Palma et al., tener este tipo de interacción podría funcionar como un factor protector al estrés que produce la alteración del rol parental y la separación con el menor ${ }^{41}$.

\section{Conclusiones}

En el presente artículo se logró identificar el nivel de estrés y las dimensiones que abarca en los padres de menores hospitalizados en las Unidades de Cuidados Intensivos Pediátricos y Neonatales, determinando que la experiencia en general es moderadamente estresante, viendo una mayor tendencia en las dimensiones de rol parental y apariencia y comportamiento del bebé. En cuanto al sexo, no se observan diferencias entre padres y madres.

A raíz de esta revisión surge la necesidad de aumentar los estudios en UCIP, debido a la escasez de investigaciones en unidades con niños y adolescentes hospitalizados. También, resulta interesante estudiar en profundidad el impacto que las brechas de los servicios públicos y privados tienen en el estrés parental, además, de cómo pueden influir las características culturales en esta situación de impacto individual y familiar.

Por otra parte, en la mayoría de las intervenciones se logra determinar que estas tienen un impacto positivo en el estrés parental, por tanto, es necesario implementar estrategias factibles que generen una disminución del estrés en los padres y por consiguiente una mejor 
recuperación de su hijo o hija, dependiendo de las distintas realidades socioculturales de los padres y los factores estructurales y ambientales de las UCI. En base a esto se pueden destacar estrategias como transformar las UCIN o UCIP en un lugar más amigable para los padres por medio de una orientación al servicio con toda la información relevante sobre la unidad, teniendo una comunicación empática, permitiendo que los padres puedan acompañar a su hijo o hija y favoreciendo su participación activa en los cuidados, según su estado de salud lo permita $27,28,29,30$. Si bien esto último puede ser algo más complejo de realizar en los servicio de salud en Chile, existen otras alternativas para implementar durante el proceso de hospitalización que generen un impacto significativo en la disminución del estrés parental, como la escritura narrativa, la cual no requiere gran cantidad tiempo, ni gastos económicos para ser aplicados.

Finalmente, es relevante mencionar que el profesional enfermero/a de cuidados críticos debe asumir como parte de sus labores el cuidado de los padres, a través de intervenciones que generen un impacto positivo en el estrés de estos. Además, es de suma importancia una mayor preparación en el equipo de enfermería para la aplicación de intervenciones que reduzcan el estrés de los padres, esto por medio de la educación permanente, con orientación y respaldo de la institución, para que se genere un cambio en la cultura organizacional y se refleje en una mayor conciencia en el equipo multidisciplinario con relación al recibimiento de los padres en la unidad y en la comprensión de sus experiencias.

Así también, las intervenciones debieran permitir que los padres puedan asumir un rol en los cuidados otorgados a su hijo o hija, lo que impactaría de forma positiva en su capacidad de participación durante la hospitalización y del proceso de salud al cual se enfrente el menor ${ }^{16}$.

\section{Referencias}

1. Astudillo A, Martínez A, Muñoz C, Pacheco M, Sepúlveda A. Acompañamiento familiar en la hospitalización del usuario pediátrico de 6 a 12 años. Cienc. enferm. [Internet]. 2012 Abr [Acceso 10 Jul 2020]; 18 (1): 67-75. Disponible en: https://scielo.conicyt.cl/scielo.php?script=sci_arttext\&pid=S0717-95532012000100007\&lng=es.

2. Hockenberry M, Wilson D, Rodgers C. Wong. Enfermería pediátrica. 10ma ed. Barcelona (Es): Elsevier; 2019. 1064 p.

3. MINSAL (Chile). Programa Nacional de Salud de la Infancia con Enfoque Integral. Santiago; 2013.

4. Selye, H. A syndrome produced by diverse noxious agents. Nature. 1936; 138, 32.

5. Ramírez M, Navarro S, Clavería C, Molina Y, Cox A. Estresores parentales en Unidad de Cuidados Intensivos Pediátricos. Rev. chil. pediatr. 2018 Abr; 89(2): 182-9.

6. Aliberch A, Miquel I. Necesidad de rol en los familiares del paciente en la unidad de cuidados intensivos. Enferm Intensiva. 2015; 26(3):101-11.

7. Raines D. Mothers stressor as the day of discharge from the NICU approaches. NANN's Journal. 2013; 13(3): 181-7.

8. Organización Mundial de la Salud (OMS). Reducción de la mortalidad en la niñez [Internet] OMS; 2020 [Actualizado Oct 2020; acceso 29 Oct 2020]. Disponible en: https://www. who.int/es/newsroom/fact-sheets/detail/children-reducing-mortality

9. Campos-Miño S, Sasbón J, Von Dessauer B. Los cuidados intensivos pediatricos en Latinoamerica. Med intensiva. [Internet]. 2011. [Acceso 29 Oct 2020]; 36(1). Disponible en: https://www.medintensiva.org/es-pdf-S0210569111002233

10. Departamento de Estadísticas e Información de Salud (DEIS). Número de defunciones por grupo de edad. Santiago de Chile; 2019.

11. Subsecretaria de Redes Asistenciales (Chile). Norma de Organización y Funcionamiento Unidades de Paciente Crítico Pediátrico. Santiago de Chile; 2017.

12. Carter M, Miles M, Buford T, Hassanein R. Parental environmental stress in pediatric intensive care units. Dimens Crit Care Nurs. 1985;4(3):180-8

13. Caruso A, Mikulic I. El estrés en padres de bebés prematuros internados en la unidad de cuidados intensivos neonatales: traducción y adaptación de la escala parental stressor scale: neonatal 
intensive care unit (PSS:NICU - M.S. Miles y D. Holditch Davis, 1987; M.S. Miles y S.G. Funk, 1998). Anu. Investig. 2012; 19(2): 19-26.

14. Tobo Medina N, Betancur Mesa P, De la Cruz Enríquez C. Estímulos, afrontamiento y adaptación en padres de recién nacidos hospitalizados en unidades de cuidado intensivo neonatal. Investig Enferm Imagen Desarr. 2017 [Acceso 30 May 2020]; 19(2):161-75. Disponible en: http://dx.doi. org/10.11144/Javeriana.ie19-2.eaap

15. Bonfill E, Lleixa M, Sáez F, Romaguera S. Estrés en los cuidados: una mirada desde el modelo de Roy. Index Enferm [Internet]. 2010 Dic [Acceso 30 May 2021]; 19(4): 279-282. Disponible en: http://scielo.isciii.es/scielo.php?script=sci_arttext\&pid=S1132-12962010000300010\&lng=es

16. Miles $M$, Funk $S$, Kasper $M$. The stress response of mothers and fathers of preterms infant. Res Nurs Health. 1992; 15: 261-9

17. Aguiñaga-Zamarripa $M L$, Reinaga-Ornelas $L$, Beltrán-Torres A. Estrés en padres de neonatos hospitalizados. Rev Enferm Inst Mex Seguro Soc. 2016; 24(1): 27-35.

18. Clarivate Analytics. Web of Science [Internet]. Clarivate Analytics; 1997 [Actualizado Nov 2020; acceso 24 Nov 2020]. Disponible https://apps.webofknowledge.com/WOS_GeneralSearch_input.do?product=WOS\&search_mode= GeneralSearch\&SID=6FduStQP4LwVtvEAPCb\&preferencesSaved=

19. Elsevier. Scopus [Internet]. Elsevier; 2004 [Actualizado Nov 2020; acceso 24 Nov 2020] Disponible en: https://www.scopus.com/search/form.uri?display=basic

20. BIREME. Biblioteca Virtual en Salud [Internet]. BIREME; 1999 [Actualizado Nov 2020; acceso 24 de noviembre] Disponible en: https://bvsalud.org/es/

21. EBSCO Industries. EBSCO [Internet]. EBSCO Industries;1984 [Actualizado Nov 2020; acceso 24 Nov 2020] Disponible en: http://web.b.ebscohost.com/ehost/search/basic?vid=1\&sid=8e20802e203d-4454-a4c1-bc28818eefe8\%40sessionmgr101

22. Fundación de apoyo a la investigación del estado de Sao Paulo (FAPESP) SciELO [Internet]. FAPESP; 1998 [Actualizado Nov 2020; acceso 24 Nov 2020] Disponible en: https://scielo.org/es/

23. BIREME. Descriptores en Ciencias de la Salud [Internet]. BIREME; 1986 [Actualizado Nov 2020; acceso 24 Nov 2020] Disponible en: http://decs2020.bvsalud.org/

24. Cano Arana, A., González Gil, T., Cabello López, J.B. por CASPe. Plantilla para ayudarte a entender un estudio cualitativo. En: CASPe. Guías CASPe de Lectura Crítica de la Literatura Médica. Alicante: CASPe; 2010. Cuaderno III. p.3-8.

25. Astudillo A, Silva $P$, Daza J. Nivel de estrés en padres de niños hospitalizados en unidades críticas pediátricas y neonatales. Cienc. enferm. [Internet]. 2019 [Acceso 06 May 2020]; 25 (18). Disponible en: https://scielo.conicyt.cl/scielo.php?script=sci_arttext\&pid=S071795532019000100214\&lng=es.

26. Betty Noergaard B; Ammentorp J; Garne E; Fenger-Gron J; Kofoed PE. Fathers' Stress in a Neonatal Intensive Care Unit. Pediatr delantero. 2018 Oct; 18 (5): 413-22.

27. Tandberg B, Flacking R, Markestad T, Grundt H, Moen A. Parent psychological wellbeing in a singlefamily room versus an open bay neonatal intensive care unit. PLoS One. [Internet] 2019 [Acceso 19 Dic 2020]; 14(11). Disponible en: https://doi.org/10.1371/journal.pone.0224488

28. Mansson C, Jakobsson U, Lundqvist P. Translation and psychometric evaluation of a Swedish version of the parental stressor scale PSS: NICU. Scand J Caring Sci. 2016; 30(1): 193-201. Disponible en: https://doi.org/10.1111/scs.12217

29. Aftyka A, Rozalska I, Rybojad B, Samardakiewicz ME. Polish version of the Parental Stressor Scale: Neonatal Intensive Care Unit. Ann Agric Environ Med. 2019; 26(1): 67-72. Disponible en: https://doi: 10.26444/aaem/89769

30. Enke C, Oliva y Hausmann A, Miedaner F, Roth B, Woopen C. Communicating With Parents In Neonatal Intensive Care Units: The Impact On Parental Stress. Patient Educ Couns. 2017; 100 (4): 710-9

31. Küçük D, Kardaş F, Güdücü F. (2017). The Effect of Spiritual Care on Stress Levels of Mothers in NICU. West. J. Nurs. Res. 2017; 40(7) 1-15.

32. Çekin $B$, Turan $T$. The stress levels of parents of premature infants and related factors in Nenoatal Intensive Care Units. Turk J Pediatr. 2018; 60(1): 117-25.

33. Kadivar M, Seyedfatemi N, Akbari N, Haghani H, Fayaz M. Evaluation of the effect of narrative writing on the stress sources of the parents of preterm neonates admitted to the NICU. J Matern 
Fetal Neonatal Med. [Internet]. 2016 [Acceso 19 Dic 2020]; 30(13). Disponible en: https://www.tandfonline.com/doi/full/10.1080/14767058.2016.1219995

34. Leong S, Lim K, Danaee M, Soh K, Lam K, Geok k, et al. The Effectiveness Of A Structured Nursing Intervention Program On Maternal Stress And Ability Among Mothers Of Premature Infants In A Neonatal Intensive Care Unit. J Clin Nurs. 2019; 28(3): 641-9

35. Akkoyun S, Tas Arslan F. Investigation Of Stress And Nursing Support In Mothers Of Preterm Infants In Neonatal Intensive Care Units. Scand J Caring. 2019; 33(2): 351-8

36. Aguiñaga-Zamarripa M, Reynaga-Ornelas $L$, Beltran-Torres A. Estrés percibido por los padres del neonato en estado crítico durante el proceso de hospitalización. Rev Enferm Inst Mex Seguro Soc. 2016; 24(1): 27-35

37. Jaciara J, Tatsch E, Maciel da Silva A, Bigolin L, Bertoldo C, Heinz da Silva J. Stress in parents of newborns in a neonatal intensive care unit. Esc Anna Nery. [Internet]. 2007 [Acceso 18 Dic 2020]; 23(1). Disponible en: http://www.scielo.br/scielo.php?script=sci_arttext\&pid=S1414$81452019000100211 \&$ lang=es

38. Balbino FS, Balieiro MMFG, Mandetta MA. Measurement of Family-centered care perception and parental stress in a neonatal unit. Rev. Latino-Am. Enfermagem. [Internet] 2016 [Acceso 03 Ene 2021]; 24(1). Disponible en: Measurement of Family-centered care perception and parental stress in a neonatal unit (nih.gov)

39. Domínguez-Martínez V, Cortés-Escárcega I. Adaptación cultural y validación de Parental Stressor Scale: Neonatal Intensive Care Unit en padres mexicanos. Enfermeria Universitaria. 2019; 16 (4): 339-349

40. Feier G, Wagner E, Almeida G, Chollopetz M. Stress experienced by mothers of preterm newborns in a neonatal intensive care unit. Rev Gaúcha Enferm. 2020; 41(1): 1-10

41. Palma E, Von Wussow F, Morales I, Cifuentes J, Ambiado S. Estrés en padres de recién nacidos hospitalizados en una unidad de paciente crítico neonatal. Rev Chil Pediatr. 2017;88(3): 332-339.

42. Sankar J, Lodha R, Kabra SK. Parental Stress in pediatric intensive care unit: how do we cope with it. Indian J pediatric. 2014; 81(11): 1141-2.

43. Doupnik S, Hill D, Palakshappa D, Worsley D, Bae H, Shaik A, Et All. Parent coping support interventions during acute pediatric hospitalizations: A meta-analysis. Pediatrics [Internet]. 2017 [Acceso 2 Ene 2021]; 140(3). Disponible en: Parent Coping Support Interventions During Acute Pediatric Hospitalizations: A Meta-Analysis | American Academy of Pediatrics (aappublications.org)

\section{Editor}

Escuela de Enfermería de la Universidad Católica Santo Toribio de Mogrovejo, Chiclayo, Perú

\section{Cómo citar este trabajo (llenado por la revista)}

Peña-Silva B, Miranda-Iglesias M, Caviedes-Fernández J, Ulloa-Ramírez V, Rementería-Rementería Y, et al. Estrés parental y sus dimensiones en Unidades de Cuidado Intensivo Neonatal - Pediátrica: Revisión Narrativa. Acc Cietna: para el cuidado de la salud [Internet]. 2021; 8(2): 67 - 84. Disponible en: https://doi.org/10.35383/cietna.v8i2.611

\section{Financiación}

El presente artículo no cuenta con financiación específica de agencias de financiamiento en los sectores público o privado para su desarrollo y/o publicación.

\section{Conflicto de interés}

Los autores del artículo declaran no tener ningún conflicto de intereses en su realización.

(C) Los autores. Este artículo es publicado por la Revista Acc Cietna: para el cuidado de la salud de la Escuela de Enfermería, Universidad Católica Santo Toribio de Mogrovejo.

Este es un artículo de acceso abierto, distribuido bajo los términos de la Licencia Creative Commons Atribución-NoComercial-CompartirIgual 4.0 Internacional (CC BY-NC-SA 4.0), que permite el uso no comercial, distribución y reproducción en cualquier medio, siempre que la obra original sea debidamente citada. 